\title{
Boundary distortions for neutral and emotional pictures
}

\author{
INGRID CANDEL, HARALD MERCKELBACH, and MAARTJE ZANDBERGEN \\ Maastricht University, Maastricht, The Netherlands
}

\begin{abstract}
In two experiments, we examined Safer, Christianson, Autry, and Österlund's (1998) claim that when emotional material is remembered, tunnel memory (i.e., the tendency to remember less of a scene than was actually shown) occurs. In Experiment 1, 81 undergraduate students drew photographs from memory after having briefly seen either four neutral or four emotional photographs. Both neutral and emotional drawings revealed boundary extension (i.e., the tendency to remember more of a scene than was actually shown). Experiment 2 relied on the camera distance paradigm (Intraub, Bender, \& Mangels, 1992). In a recognition test, 60 undergraduate students judged the camera distance of previously seen neutral or emotional photographs. The majority of them demonstrated accurate judgments and neither extended nor restricted picture boundaries. Those participants who made an error more often displayed a boundary extension than a tunnel memory error. Taken together, our results suggest that boundary extension for neutral and emotional photographs is a more robust phenomenon than its counterpart, tunnel memory.
\end{abstract}

Over the past few years, memory illusions have been the object of numerous studies (for a review, see Schacter, 1999). One type of illusion that occurs when people try to remember neutral photographs is known as the boundary extension phenomenon. This phenomenon refers to the tendency to remember information that was not in the picture but that was likely to have existed just outside the camera's field of view (Intraub \& Richardson, 1989). Evidence for this distortion has been obtained with a wide variety of memory tests. For example, when participants are exposed to neutral photographs and then asked to draw these photographs as accurately as possible from memory, their drawings tend to contain a greater proportion of background than was actually shown (Intraub \& Bodamer, 1993; Intraub \& Richardson, 1989). Extended boundaries can also be demonstrated with recognition tests (e.g., the camera distance paradigm; Intraub, Bender, \& Mangels, 1992; Intraub \& Bodamer, 1993; Nyström, 1993). Typically, following exposure to neutral photographs, participants are confronted with test photographs. They are asked to judge whether the test photographs are the same, closer up, or farther away, as compared with the originals. In general, participants tend to accept wide-angle distractors as copies of the originals, indicating that they remember more of the scene than was actually shown. Nyström found evidence for boundary extension when he asked participants to

The authors are grateful to Helene Intraub for her helpful comments on an earlier draft of this manuscript. Correspondence concerning this article should be addressed to I. Candel, Department of Experimental Psychology, Maastricht University, P. O. Box 616,6200 MD, Maastricht, The Netherlands (e-mail: i.candel@psychology.unimaas.nl). move-physically — boundaries of test pictures in order to match their recollections of the original photographs.

Although boundary extension is a robust phenomenon, it appears to have some constraints. For example, the amount of extension is maximal when the original pictures are close-ups. The degree of boundary extension decreases as original photographs become more wideangled, and at some point, no distortion occurs. Another limiting condition is time. With the passage of time between exposure to original photographs and reproduction during a subsequent test, boundary extension disappears (e.g., Intraub et al., 1992).

Intraub and her colleagues (e.g., Intraub, Gottesman, $\&$ Bills, 1998) argued that boundary extension is the result of activation of an automatic perceptual schema. In the words of Gottesman and Intraub (1999), "When we view a picture of a scene, the visual system rapidly and automatically extrapolates beyond the borders of that view. Boundary extension is thought to occur because the expected regions just outside a picture's borders are so integral to comprehension of the picture that the expected information becomes incorporated in the viewer's mental representation" (p. 86).

There is a paucity of experimental work addressing representations of emotional photographs. One exception is the study by Safer, Christianson, Autry, and Österlund (1998). These authors argued that when emotionally arousing photographs are viewed, attention automatically narrows to details that constitute the source of the emotional arousal (i.e., the attentional-narrowing hypothesis; Christianson, 1992). This would result in what the authors call tunnel memory, which refers to poor memory for information in the mental periphery and a relatively strong mnemonic representation of critical and 
central details. The experiments by Safer et al. (1998) provide some support for such a pattern. In their experiments, participants saw a series of photographs that formed a story line. Half of the participants were shown a neutral story, whereas the other half saw an aversive story. The two stories differed with regard to two target photographs. During the recognition test of Experiment 4 , test pictures of the target scenes were presented, but they had been photographed with different focal lengths (i.e., the camera distance paradigm; Intraub et al., 1992). The participants had to judge whether the camera distance was much closer than, slightly closer than, identical to, slightly farther than, or much farther than the original. They tended to accept a more close-up version of the emotional target (i.e., tunnel memory) and a more wide-angle version (i.e., boundary extension) of the corresponding neutral target. However, in their Experiment 3, Safer et al. found the opposite pattern. During the recognition phase of that experiment, the participants evaluated test photographs that were exact copies of the targets. They were led to believe that the test stimuli had been photographed with different focal lengths. For the first test picture, the participants were quite accurate in recognizing that the camera distance was the same as that of the original target. That is, no boundary distortion occurred for neutral or emotional material. However, for the second test picture, both the neutral and the emotional groups displayed boundary extension. Taken together, these results suggest that under some conditions that are not well understood, tunnel memory for negative material might occur. Boundary distortions in remembering emotionally negative photographs therefore warrants further study. The present experiments addressed this issue by using two different methods for testing memory for negative photographs. Experiment 1 relied on a drawing task, whereas Experiment 2 employed the camera distance paradigm.

\section{EXPERIMENT 1}

Boundary distortions for emotional material have not been examined with what appears to be one of the most straightforward and reliable methods for studying such distortions-namely, the drawing task described by Intraub and Richardson (1989) and Intraub and Bodamer (1993). With this in mind, we conducted Experiment 1. On the basis of Safer et al.'s (1998) findings (Experiment 4), we expected that tunnel memory (boundary restriction) would occur for drawings of aversive photographs, whereas boundary extension would occur for drawings of neutral material.

\section{Method}

Participants. The participants were 81 Maastricht University undergraduates (60 women). Their mean age was 20 years $(S D=$ 1.33; range, 18-23 years). The participants were tested individually. They received a small financial compensation for their participation.

Materials. There were two types of stimuli-namely, neutral and emotional photographs. Neutral photographs used in previous studies by Intraub and her colleagues (e.g., Intraub \& Berkowits, 1996; Intraub, Gottesman, Willey, \& Zuk, 1996) were downloaded from the Web. ${ }^{1}$ Emotional photographs were drawn from the International Affective Picture System (Lang, Bradley, \& Cuthbert, 1995). For this experiment, we selected four aversive photographs. Like the neutral photographs, they depicted single objects (i.e., a decomposed dog, a wounded hand, a wounded face, and a gun) against a simple background. In the normative sample of Lang et al. (1995), the aversive targets used in the present experiment were rated as highly unpleasant and highly arousing. The amount of space occupied by objects was exactly the same for all the photographs. So, the potential for extension or restriction was similar for the two types of photographs. The photographs measured $100 \times$ $150 \mathrm{~mm}$ (approximately $4 \times 6$ in.) and were presented on a computer screen with a gray background.

Design and Procedure. The participants were assigned to either the neutral $(n=42)$ or the emotional $(n=39)$ condition. In both conditions, the participants saw four photographs for $15 \mathrm{sec}$ each, after they had read the following instructions (Gottesman \& Intraub, 1999, p. 90):

You will be presented with 4 photographs, for $15 \mathrm{sec}$ each. Please pay close attention to each picture and try to remember the main object and the background in as much detail as possible. Try to remember everything in the picture, including its layout. That is, the size and location of everything in the picture space. In other words, try to retain an exact copy of each picture in your memory.

Photograph sequence was counterbalanced, and presentation of the photographs was followed by a drawing test. Response sheets were handed out in the same order as the picture presentation. The instructions for this task were taken from Gottesman and Intraub (1999, p. 90) and were as follows:

In each rectangle, draw the picture named above in as much detail as possible. Don't worry if you are not a great artist; just do your best to represent everything you saw in the picture. Consider the edges of the rectangle to be the edges of the picture you saw. Try to capture the layout of the picture. That is, try to draw everything in the same relative size and position as in the picture. After you draw each picture, make all the changes you think are necessary, and if you want to clarify any part of your drawing please feel free to use words as labels.

Following the drawing test, the participants were asked to rate the photographs on 9-point scales in terms of experienced pleasure, arousal, and threat (anchors: $1=$ not at all, $9=$ very much).

\section{Results and Discussion}

Manipulation check. The emotional photographs were rated as less pleasant than were the neutral photographs $[t(78)=15.09, p<.001]$, the means being 2.75 $(S D=0.82)$ and $5.74(S D=0.94)$, respectively. Moreover, the emotional photographs were rated as more arousing than were the neutral photographs $[t(78)=$ $-7.39, p<.001]$, the means being $5.97(S D=1.44)$ and $3.38(S D=1.68)$, respectively. Similarly, the emotional photographs were evaluated as more threatening than were the neutral photographs $[t(78)=-8.88, p<.001]$, the means being $4.99(S D=1.75)$ and $2.10(S D=1.12)$, respectively.

Drawings. Due to technical problems, 1 participant's data were lost, leaving 80 participants for analysis. A total of 320 drawings (168 neutral and 152 emotional drawings) were obtained. All the drawings were made within $100 \times 150 \mathrm{~mm}$ (approximately $4 \times 6$ in.) rectangles. To measure the area of the pertinent object, we used the paper-and-pencil method described by Gottesman and Intraub (1999). Thus, all the drawings were covered 
with a transparent graph paper $\left(5 \mathrm{~mm}^{2}\right)$. Next, an independent judge counted the number of grid squares that each object covered and divided this by the number of grid squares that the object in the original stimulus covered. A proportion of one indicated that the object's size was accurately captured in the drawing. A proportion smaller than one indicated boundary extension (i.e., the object covered a smaller area in the drawing than in the original). A proportion exceeding one indicated tunnel memory (i.e., the object covered a larger area in the picture space). Proportions ( proportions drawn; Intraub \& Bodamer, 1993) were averaged across drawings of both categories (see Table 1). The mean proportion drawn was significantly smaller than 1.00 for both neutral $[t(41)=$ $21.21, p<.001]$ and emotional $[t(37)=16.22, p<.001]$ objects, indicating that the participants displayed extended boundaries for both the neutral and the emotional photographs. The amount of extension did not differ between the two picture categories $[t(78)=1.2, p=.23]$. Figure 1 shows an example of boundary extension. The tendency to extend boundaries was typical not only for proportions averaged over photographs. The proportion drawn was less than 1.00 for all individual drawings of both categories, except for five neutral drawings (three for tire, one for bananas, and one for bucket) and three emotional drawings (two for wounded hand and one for wounded face).

The main results of Experiment 1 can be summarized as follows. To begin with, our findings provide a straightforward replication of earlier work by Intraub and associates (e.g., Intraub et al., 1992; Intraub \& Bodamer, 1993; Intraub et al., 1996; Intraub \& Richardson, 1989), in the sense that clear boundary extension occurred for neutral photographs. Second, in contrast to the tunnel memory phenomenon (i.e., boundary restriction) for aversive stimuli obtained by Safer et al. (1998) in their Experiment 4, our participants also extended boundaries for emotional photographs. Note that our findings are very similar to those found in Safer et al.'s Experiment 3. The present findings seem to replicate those of Intraub and co-workers (e.g., Intraub et al., 1992; Intraub \& Bodamer, 1993; Intraub \& Richardson, 1989) in showing that boundary extension occurs when

Table 1

Mean Proportion Drawn for Each Photograph and Averaged for Neutral and Emotional Stimuli (With Standard Deviations)

\begin{tabular}{clll}
\hline \multirow{2}{*}{ Stimuli } & \multicolumn{1}{c}{ Picture } & \multicolumn{2}{c}{ Proportion Drawn } \\
\cline { 2 - 4 } Neutral & bear & $M$ & $S D$ \\
& bananas & .41 & .17 \\
& bucket & .41 & .25 \\
& tire & .45 & .23 \\
$M$ & & .57 & .25 \\
Emotional & decomposed dog & .46 & .14 \\
& wounded hand & .49 & .16 \\
& wounded face & .34 & .27 \\
$M$ & gun & .43 & .22 \\
& & .42 & .16 \\
\hline
\end{tabular}

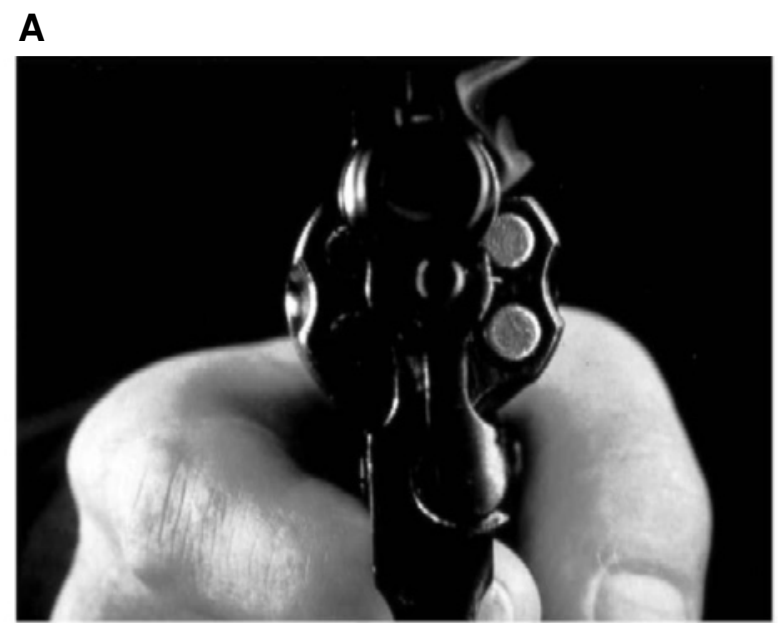

B

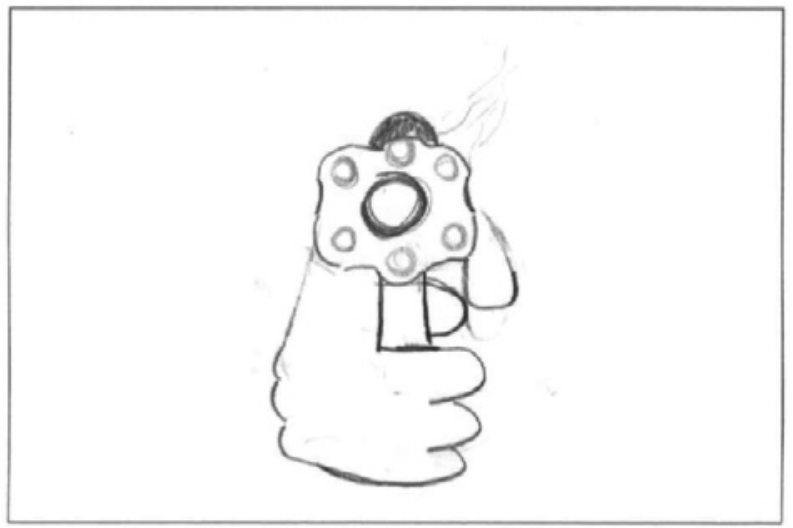

Figure 1. Examples of (A) an emotional photograph and (B) a drawing showing boundary extension.

single neutral photographs are remembered. Moreover, our results indicate that the same memory illusion occurs for emotional photographs. The perceptual schema hypothesis proposed by Intraub et al. (1998) offers the most parsimonious account for these results. Apparently, photographs activate perceptual schemata irrespective of their emotional valence. The information implicated by the schema is then incorporated into memory. Consequently, in remembering photographs, more background is assumed than was actually present.

\section{EXPERIMENT 2}

The findings obtained in our first experiment are difficult to reconcile with those of Safer et al. (1998), who reported tunnel memory for emotional photographs in their Experiment 4. One reason for this discrepancy might be that the present study relied on isolated photographs, whereas Safer et al. made use of photographs that formed a story line. Perhaps, then, stories yield a schema that is superimposed on schemas for separate photographs. Such a higher order schema might give rise 
to tunnel memory, especially when a story is emotionally compelling. In Experiment 2, we tested whether tunnel memory would occur when emotional photographs formed a story line. Furthermore, we adopted the camera distance paradigm described by Intraub et al. (1992) to assess boundary distortions in memory for neutral and matched emotional photographs.

\section{Method}

Participants. The participants were 60 Maastricht University undergraduates (44 women). Their mean age was 19 years $(S D=1.25$; range, 18-23 years). The participants were tested individually. They received a small financial compensation for their participation.

Materials. Two series of color photographs were used as stimulus material. The series consisted of three phases that formed a storyline. The storyline was inspired by that of Safer et al. (1998). The first phase of the neutral series showed a man arriving in his car at a house, where a woman opens the front door (four photographs). The second and critical phase consisted of two photographs (Targets 1 and 2): one a photograph of the man giving the woman a present and the other of the woman smiling. The third phase (three photographs) showed the man leaving by car. The emotional series was identical to the neutral series with respect to the first and the third phases. The second phase of the emotional series also consisted of two photographs (Targets 1 and 2). However, here the first target showed the man threatening the woman with a knife, and the second target showed the woman bleeding from a wound in her neck. Neutral and emotional pictures for all phases were photographed from identical distances and angles.

Design and Procedure. The participants were assigned to either the neutral $(n=30)$ or the emotional $(n=30)$ condition. In both conditions, the participants saw nine photographs for $5 \mathrm{sec}$ each. The photographs were presented on a computer screen with a gray background. The presentation was followed by a 7-min filler task that involved rating the story in terms of emotionality on a $100-\mathrm{mm}$ Visual Analog Scale $(0=$ not emotional at all, $100=$ extremely emotional) and answering questionnaires. Next, the participants were given the camera distance instructions. They were told that they would see some of the photographs again but that scenes might now be photographed from different camera distances. In fact, the test photographs were exact copies of the original targets. The participants were asked to judge the camera distance on a 3-point scale: $-1=$ farther than original, $0=$ no change, and $+1=$ closer than original. They were given a practice item with an unrelated neutral photograph to make sure that they understood the instructions. Next, the two targets were presented for $15 \mathrm{sec}$ each.

\section{Results and Discussion}

Manipulation check. The emotional story was rated as more emotional than the neutral story $[t(58)=-3.49$, $p<.01]$, means being $42.10(S D=22.49)$ and 21.63 $(S D=22.99)$, respectively.

Recognition test. Chi-square tests (group $\times$ judgments) yielded no significant differences between the neutral and the emotional conditions (all $p \mathrm{~s}>.41$; Pearson chi-square, likelihood ratio, linear by linear), indicating that, in both groups, similar proportions of participants endorsed the three answer options. Moreover, as can be seen in Table 2, the large majority of the participants in both groups accurately recognized that the test pictures were exact copies of the targets. Accordingly, in both groups, mean scores for Targets 1 and 2 did not differ significantly from zero (no change; all $t \mathrm{~s}<1.0$,
Table 2

Frequency and Percentage of Participants That Endorsed Each Camera Distance Option for the Two Target Photographs

\begin{tabular}{|c|c|c|c|c|c|c|c|c|}
\hline \multirow[b]{3}{*}{ Option } & \multicolumn{4}{|c|}{ Neutral $(n=30)$} & \multicolumn{4}{|c|}{ Emotional $(n=30)$} \\
\hline & \multicolumn{2}{|c|}{ Target 1} & \multicolumn{2}{|c|}{ Target 2} & \multicolumn{2}{|c|}{ Target 1} & \multicolumn{2}{|c|}{ Target 2} \\
\hline & Freq. & $\%$ & Freq. & $\%$ & Freq. & $\%$ & Freq. & $\%$ \\
\hline Farther & 2 & 7 & 4 & 13 & 2 & 7 & 2 & 7 \\
\hline No change & 20 & 67 & 23 & 77 & 21 & 70 & 24 & 80 \\
\hline Closer & 8 & 26 & 3 & 10 & 7 & 23 & 4 & 13 \\
\hline
\end{tabular}

all $p$ s $>.05)$. Hence, neither group showed boundary extension or boundary restriction. However, those individuals who did endorse an incorrect option for Target 1 displayed boundary extension (closer) significantly more often than tunnel memory [ farther; $\chi^{2}(1)=6.37, p<$ .01]. No such difference was found for Target $2\left[\chi^{2}(1)=\right.$ $0.74, p=.39]$.

The main results of Experiment 2 can be summarized as follows. To begin with, the participants were quite accurate in recognizing that the boundaries of the test stimuli in the camera distance task were the same as those of the original targets. In addition, for the first, but not for the second, target, individuals who gave an incorrect camera distance judgment displayed a tendency to extend boundaries, irrespective of the emotional valence of the targets. To a certain degree these results resemble those found in Safer et al.'s (1998) Experiment 3. In that experiment, the authors also failed to obtain tunnel memory or boundary extension for the neutral or the emotional Target 1 . Moreover, contrary to their prediction, both groups extended boundaries for Target 2 .

\section{GENERAL DISCUSSION}

With two different sets of stimuli and two different memory tests, the findings of our experiments do not support Safer et al.'s (1998) claim that, as compared with neutral scenes, aversive scenes are remembered in a tunnellike fashion. More specifically, in the free-recall drawing task of Experiment 1, the participants extended, rather than restricted, boundaries for neutral as well as for emotional photographs, indicating that they remembered a wide-angle version of both types of targets. In Experiment 2 , the majority of the participants in the neutral, as well as in the emotional, condition were quite accurate in recognizing that the test stimuli were exact copies of the targets. As well, those participants who did display a boundary distortion for Target 1 extended, rather than restricted, photograph boundaries.

The present results demonstrate that when boundary distortions occur in memory for neutral or emotional pictures, they are likely to be extensions, rather than the restrictions implicated by the concept of tunnel memory. Thus, our findings support Intraub's (e.g., Intraub et al., 1998) view that when remembering scenes, people rely on a perceptual schema that elicits extension tendencies. Perhaps, then, boundary extension is immune to emo- 
tional content because it involves early perceptual processes, rather than relatively late reconstructive memory processes.

At first sight, boundary extension for emotional material appears to contradict the well-documented phenomenon of weapon focus (Steblay, 1992). Weapon focus is defined by good memory for central details and poor memory for peripheral details. However, it should be stressed that boundary extension refers to proportional relations between central and peripheral details, and not to their accuracy per se. That is to say, a highly extended representation is defined by reduced central details and enlarged peripheral details. Such a pattern does not necessarily imply an inaccurate memory for central details. Meanwhile, commission and/or omission errors in peripheral information may accompany boundary extension (for an example, see Intraub \& Richardson, 1989), and precisely these errors may also occur as byproducts of the weapon focus phenomenon (Steblay, 1992). Boundary extension and weapon focus are, therefore, not necessarily rival phenomena.

How do our findings relate to those of Safer et al. (1998)? In our view, the data presented by Safer et al. are far less convincing than the title of their study suggests. Straightforward tunnel memory for emotional material did show up only in one of their experiments (i.e., in Experiment 4), whereas boundary extension for emotional material was found in one of their other experiments (i.e., in Experiment 3). On the basis of their findings and the present results, we would argue that boundary extension is a more common phenomenon than tunnel memory. It appears that the latter phenomenon occurs only under specific conditions. Interestingly, the experiment in which Safer et al. obtained a tunnel memory effect relied on a recognition test in which participants evaluated test stimuli that deviated from the original targets. It is possible that tunnel memory critically depends on this paradigm. However, if this were to be the case, the question would arise as to whether tunnel memory tells us something about the features of emotional memory or about confusion created by exposure to deviant test stimuli. Clearly, this issue requires further study.

\section{REFERENCES}

Christianson, S.- $\AA$. (1992). Emotional stress and eyewitness memory: A critical review. Psychological Bulletin, 112, 284-309.

Gottesman, C. V., \& Intraub, H. (1999). Wide-angle memories of close-up scenes: A demonstration of boundary extension. Behavior Research Methods, Instruments, \& Computers, 31, 86-93.

Intraub, H., Bender, R. S., \& Mangels, J. A. (1992). Looking at pictures but remembering scenes. Journal of Experimental Psychology: Learning, Memory, \& Cognition, 18, 180-191.

Intraub, H., \& Berkowits, D. (1996). Beyond the edges of a picture. American Journal of Psychology, 109, 581-598.

Intraub, H., \& Bodamer, J. L. (1993). Boundary extension: Fundamental aspect of pictorial representation or encoding artifact? Journal of Experimental Psychology: Learning, Memory, \& Cognition, 19, 1387-1397.

Intraub, H., Gottesman, C. V., \& Bills, A. J. (1998). Effects of perceiving and imaging scenes on memory for pictures. Journal of Experimental Psychology: Learning, Memory, \& Cognition, 24, 186201.

Intraub, H., Gottesman, C. V., Willey, E. V., \& Zuk, I. J. (1996). Boundary extension for briefly glimpsed photographs: Do common perceptual processes result in unexpected memory distortions? Journal of Memory \& Language, 35, 118-134.

Intraub, H., \& Richardson, M. (1989). Wide-angle memories of close-up scenes. Journal of Experimental Psychology: Learning, Memory, \& Cognition, 15, 179-187.

LANG, P. J., Bradley, M. M., \& CUthbert, B. N. (1995). International affective picture system (IAPS): Technical manualand affective ratings. Gainesville: University of Florida, Center for Research in Psychophysiology.

Nyström, M. (1993). Is picture memory wide-angle? Psychological Research Bulletin, 33, 1-16.

Safer, M. A., Christianson, S.-Å, Autry, M. W., \& Österlund, K. (1998). Tunnel memory for traumatic events. Applied Cognitive Psychology, 12, 99-117.

SCHACTER, D. L. (1999). The seven sins of memory: Insights from psychology and cognitive neuroscience. American Psychologist, 54, 182-203.

Steblay, N. M. (1992). A meta-analytic review of the weapon focus effect. Law \& Human Behavior, 16, 413-424.

\section{NOTE}

1. http://faculty-staff.ou.edu/G/Carmela.Gottesman-1/web page exp.html.

(Manuscript received July 17, 2001; revision accepted for publication May 31, 2002.) 